\title{
Isolation of Tributyltin Chloride Resistance Bacteria and Rapid Electrochemical Determination of Bacterial Organotin Degradation Activity
}

\author{
Bong Geun Jeong, Seok Won Hong, ${ }^{\dagger}$ Yong Su Choi, ${ }^{\dagger}$ Rangarajulu Senthil Kumaran, Mia Kim, ${ }^{\S}$ \\ Sunghyun Kim, ${ }^{*}$ and Hyung Joo Kim*
}

\author{
Department of Microbial Engineering, Konkuk University, Seoul 143-701, Korea. E-mail: hyungkim@konkuk.ac.kr \\ ${ }^{\dagger}$ Water Environment Center, Korea Institute of Science and Technology, P.O. Box 131, Cheongryang, Seoul 130-650, Korea \\ ${ }^{\ddagger}$ Department of Bioscience and Biotechnology, Konkuk University, Seoul 143-701, Korea \\ ${ }^{\S}$ Department of Microbiology, Pusan National University, Busan 609-735, Korea \\ Received October 6, 2010, Accepted November 16, 2010
}

Key Words: TBT, Tributyltin, Electrochemical TBT analysis, TBT resistance bacteria

\begin{abstract}
Organotin compounds including tributyltin chloride (TBT) have been extensively employed in a variety of industrial products, such as antifouling paints for boat, wood preservatives, biocides, and plastic stabilizers. ${ }^{1}$ Among the organotin compounds, a great deal of research has indicated that TBT is the most toxic compound known to aquatic ecosystems. ${ }^{1,2}$ The widespread use of TBT, therefore, has resulted in serious environmental problems around the world, including endocrine disruptions of aquatic life. Organotins including TBT in aqueous samples are generally evaluated via gas chromatography with flame photometric detection (GC-FPD). ${ }^{3}$ The principal drawbacks associated with these methods are the time required for sample pretreatment and for the steps of organotin extraction with various solvents. Although these methods do have a certain degree of precision, the relevant instrumentations are quite costly, and require careful maintenance. Therefore, the lack of simple and rapid methods for the analysis of tin compounds in aqueous samples is something of a common problem in research into the microbial degradation of organotins.

Several previous inquiries have demonstrated that the polarographic reduction peak of tin is enhanced by the adsorption of its complexes to tropolone on the electrode surface. ${ }^{4,5,6}$ This effect has been applied in the development of simpler voltammetric procedures, such as adsorptive stripping voltammetry (ASV) using a glassy carbon mercury film electrode to determine tin concentrations in water and pharmaceutical samples.

In this study, TBT resistance bacteria were isolated from a variety of sediments and aqueous samples using TBT-containing media. The isolated bacteria were identified and their TBTdegrading activity was initially evaluated via a rapid electrochemical tin analysis method based on the ASV. In an effort to confirm the efficacy of the electrochemical method, the GCFPD technique was also applied for the validation of TBT concentration variations in bacterial media. This electrochemical approach may represent a simple, rapid, and unique research method for the investigation of organotin compounds in the ecosystem and bacterial TBT degradation.
\end{abstract}

\section{Experimental}

Chemicals. Analytical-grade chemicals were used for all studies. TBT (tributyltin chloride, purity, purity: 96\%), DBT (dibutyltin dichloride, purity: 96\%), MBT (monobutyltin trichlo- ride, purity: 96\%) were acquired from Sigma-Aldrich Chemicals (USA). The organotin stock solutions were prepared in methanol and stored at $-20{ }^{\circ} \mathrm{C}$.

Isolation and Identification of TBT-resistant Bacteria. To isolate TBT-resistant bacteria, more than 300 sediment samples were collected from various industry complex and shipbuilding yards near the Ulsan area of South Korea. ${ }^{1,7}$ The samples were obtained using an Eckman dredge, then scraped into sterilized polycarbonate centrifuge bottles. The sample was vigorously stirred with a vortex mixer and allowed to stand for $15 \mathrm{~min}$. The supernatant was employed as the source of bacteria for isolation. To prepare the enrichment cultures, the sample supernatants were inoculated in nutrient broth (NB) containing TBT $(50 \mathrm{mg} / \mathrm{L})$, then incubated at $30{ }^{\circ} \mathrm{C}$. The enrichment cultures were prepared via two cycles of culture for one week per sample. Nutrient agar ( $\mathrm{pH} 7.0$ ) containing various concentrations of TBT was used as a basic isolation medium. Appropriate amounts of TBT stock solutions were added to autoclaved nutrient broth that had cooled to $50{ }^{\circ} \mathrm{C}$. The final TBT concentrations in the nutrient media were maintained at $50 \mathrm{mg} / \mathrm{L}$. Using the solid nutrient agar containing TBT, morphologically different colonies were isolated. The isolated bacteria were cultivated in liquid media containing TBT for further experiments. ${ }^{7}$ The selected bacterial strains were identified via $16 \mathrm{~S}$ rRNA analysis. All processes of the $16 \mathrm{~S}$ rRNA analysis were conducted by a custom 16S rRNA analysis service (Macrogen Inc. Seoul, Korea).

Sample Preparation for Tin Analysis. After 30 hours of growth in a shaking incubator at $30{ }^{\circ} \mathrm{C}$, the culture supernatants were collected via centrifugation (3000 rpm) of the bacterial culture for 20 minutes at $4{ }^{\circ} \mathrm{C}$. Each supernatant was then filtered with a disposable filter $(0.2 \mu \mathrm{m}$ in pore size $)$ to remove bacterial cell debris. For experiments involving the use of ASV, the filtered culture supernatants were UV-irradiated for $3 \mathrm{~h}$ (in quartz tubes, by a $1 \mathrm{kw}$ mercury vapor lamp) to eliminate interference caused by organic materials. The samples were then filtered with acid-washed filters $(0.45 \mu \mathrm{m}$ Nucleopore, Korea Co., Korea) and acidified to $\mathrm{pH} 2.8$ with $2 \mathrm{M} \mathrm{HCl}^{5}$

Electrochemical Tin Analysis Using ASV. The tin concentration in the bacterial medium was analyzed using adsorptive stripping voltammetry. A computer-controlled potentiostat (CHI-600C, CH Instruments, USA) was employed in the study. A Teflon covered glassy-carbon cylinder (diameter $7.04 \mathrm{~mm}$, 
Cypress Systems, USA), a platinum wire (surface area: 31.61 $\mathrm{cm}^{2}$ ), and an $\mathrm{Ag} / \mathrm{AgCl}$ electrode (RE-5B, Bioanalytical Systems, USA) were used as a working electrode, counter-electrode and reference electrode, respectively. The measurement system using ASV, which consisted of a conventional three-electrode system, is depicted in Fig. 1. The electrodes were immersed into an electrochemical cell containing $20 \mathrm{~mL}$ of sample solution, $0.4 \mathrm{~mL}$ of $1 \mathrm{~g} / \mathrm{L} \mathrm{HgCl}_{2}$ solution, and $0.2 \mathrm{~mL}$ of $0.4 \mathrm{mM}$ tropolone solution. The deposition potential $(-0.4 \mathrm{~V})$ was applied to the glassy-carbon electrode while the solution was stirred. Following the pre-concentration step (usually $10 \mathrm{~min}$ ), the stirring was halted, and after $20 \mathrm{sec}$, the voltammogram was recorded via the application of a negative-going square-wave voltammetric potential scan (with a frequency of $20 \mathrm{~Hz}$, amplitude of $25 \mathrm{mV}$, potential step of $5 \mathrm{mV}$, and scan termination at $-0.85 \mathrm{~V})$. Electrochemical experiments were conducted at $25^{\circ} \mathrm{C}$. The electrochemical results from the ASV analysis were then compared via the GC-FPD method. All experiments were repeated at least three times.

Analysis of Organotins Using GC-FPD. The organotins were extracted from bacteria culture supernatants and analyzed as previously described. ${ }^{3,7}$ In brief, $20 \mathrm{~mL}$ of bacterial supernatant was extracted twice with $10 \mathrm{~mL}$ of $0.1 \%$ tropolone-benzene solution (w/v) after the addition of $5 \mathrm{~mL}$ of $1 \mathrm{~N} \mathrm{HCl}$ solution. The organic layer was dried with anhydrous $\mathrm{Na}_{2} \mathrm{SO}_{4}$ and concentrated to $1 \mathrm{~mL}$ with a rotary evaporator. After propylation with $2 \mathrm{~mL}$ of $n$-propyl magnesium bromide, $5 \mathrm{~mL}$ of $1 \mathrm{~N} \mathrm{H}_{2} \mathrm{SO}_{4}$ was added in order to destroy any excess Grignard reagent. Distilled water $(50 \mathrm{~mL})$ was then added to the mixtures and the organotins were extracted twice with $10 \mathrm{~mL}$ of $10 \%$ benzenehexane solution. After concentration to $1 \mathrm{~mL}$ with a rotary evaporator, the mixtures were cleaned with Sep-Pak Plus florisil cartridge columns (Waters Korea Co., Korea). The filtrates were subsequently injected into a GC-FPD (Model 3300, Varian, Co., USA) for further analysis. A capillary column coated with polydimethylsiloxane (inner diameter $0.25 \mathrm{~mm}$, length $30 \mathrm{~m}$, film thickness $0.25 \mu \mathrm{m}, \mathrm{J} \& \mathrm{~W}$ Scientific, USA) was employed for GC separation. The operating conditions were as follows: injector temperature $225^{\circ} \mathrm{C}$, detector temperature $250{ }^{\circ} \mathrm{C}$, column temperature programmed from $50{ }^{\circ} \mathrm{C}$ (for $2 \mathrm{~min}$ for initial temperature) to $240{ }^{\circ} \mathrm{C}$ (for $10 \mathrm{~min}$ final temperature) at a rate of $30^{\circ} \mathrm{C} / \mathrm{min}$. The detector was operated with a $610-\mathrm{nm}$ optical filter and an air/hydrogen flame. Nitrogen (flow rate: $35 \mathrm{~mL} /$ $\min$ ) was used as the carrier gas.

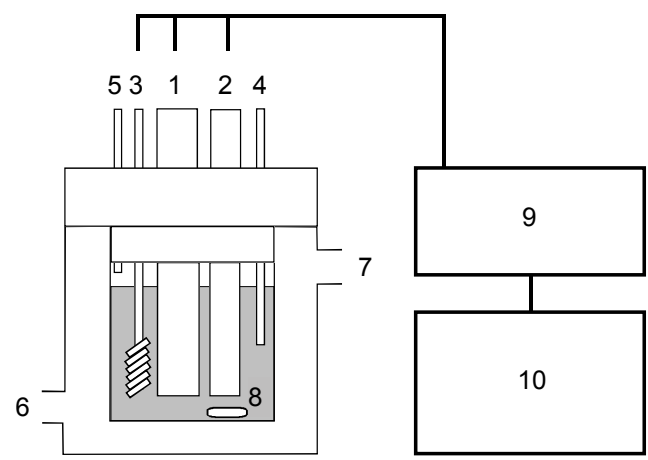

Figure 1. Schematic diagram of the TBTC measurement system using ACSV. (1) Working electrode, (2) Reference electrode, (3) Counter electrode, (4) $\mathrm{N}_{2}$ inlet, (5) $\mathrm{N}_{2}$ outlet, (6) Water inlet, (7) Water outlet, (8) Magnetic stirrer, (9) Potentiostat, (10) PC for data acquisition.

\section{Results and Discussion}

Isolation and Identification of TBT-resistant Bacteria. The colonies grown in the solid medium containing TBT $(50 \mathrm{mg} / \mathrm{L})$ were obtained after the enrichment culture with 300 samples obtained from the shipyard. Among the 300 collected samples, microbial growth was observed at the 96 samples (TBT resistance bacterial growth possibility per collected sample: $32 \%$ ). Only five morphologically different bacterial strains were isolated from the solid cultures, and designated as TDB-1 to TDB-5. The 16S rRNA analysis identified the isolates (TDB-1 to TDB-5) as Klebsiella pneumoniae, Klebsiella sp., Enterobacter agglomerans, Pantoea sp., and Alcaligenes sp., respectively. When the TBT-resistance ability of the isolated strains was evaluated in nutrient broth (in an Erlenmeyer flask) containing different concentrations of TBT $(0-55 \mathrm{mg} / \mathrm{L}$ of TBT $)$, the growth of the isolated strains was inversely related to the concentration of TBT in the culture medium (Fig. 2). Among them, strain TDB-1 (identified as Klebsiella pneumoniae) evidenced the highest growth yield in the presence of considerable amounts of TBT. According to our results, the presence of TBT in the medium profoundly inhibits bacterial growth over the tested range of concentrations. Many previous studies have shown TBT to be the most inhibitory organotin compound toward bacterial growth, particularly for respiratory systems. ${ }^{1,7,8,9}$ Therefore, it is plausible to conclude that the presence of TBT in the medium resulted in the isolation of a very small proportion of the resistant strain isolation from more than 300 sediment samples. The experimental results also demonstrated that the toxicity of TBT is speciesdependent. Among the isolates, TDB-1 (identified as Klebsiella pneumonia) which evidenced the highest degree of TBT resistance activity, was selected for further experiments in this work.

ASV for Bacterial Organotin Degradation Analysis. Typical traces of the current change owing to the reduction of tin at the electrode surface with different concentrations of TBT in the fresh medium are shown in Fig. 3A. The general form of reduction current to the presence of tin was a reverse rise in current as a function of potential decrease, declining when potentials in the range of -0.7 to $-0.8 \mathrm{~V}$ were reached. The maximum reduction current levels varied with the concentration of tin (i.e. TBT in the medium) fed to the measurement system. The peaks derived in this study, however, appeared at a more

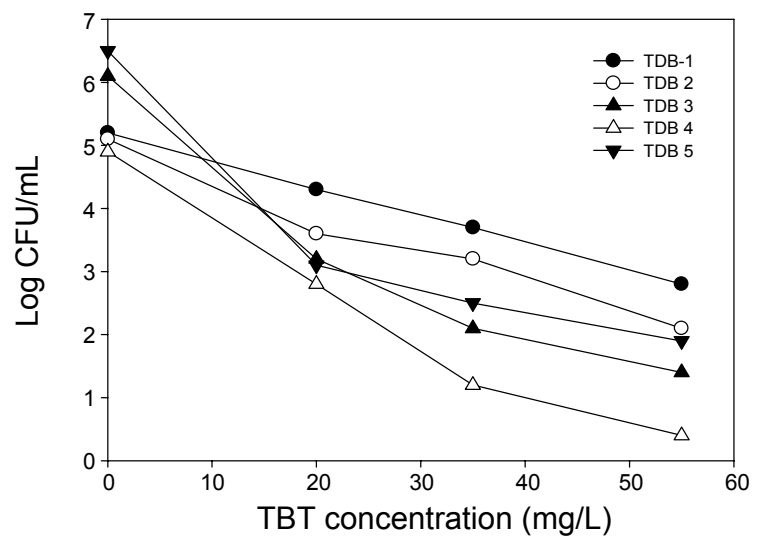

Figure 2. CFU (colony forming unit) of the isolated bacteria in nutrient broth culture medium containing different concentrations of TBT. Cultivations were conducted for $30 \mathrm{hr}$ at $30^{\circ} \mathrm{C}$ using a rotary shaker. 
(A)

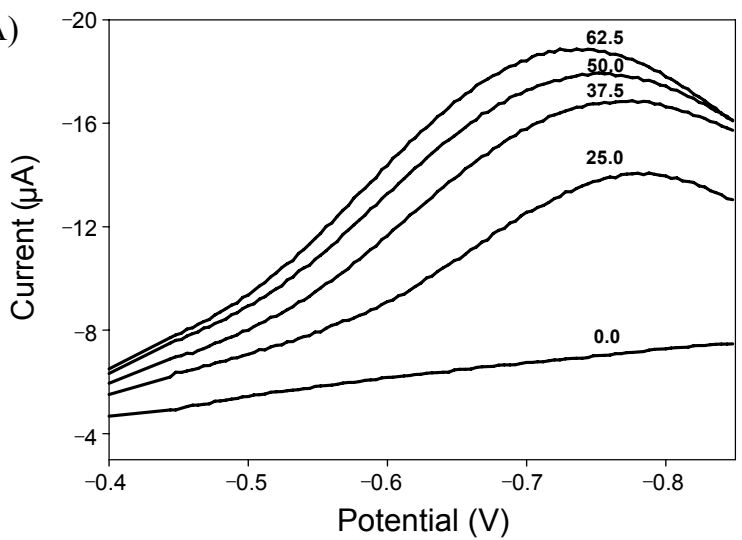

(B)

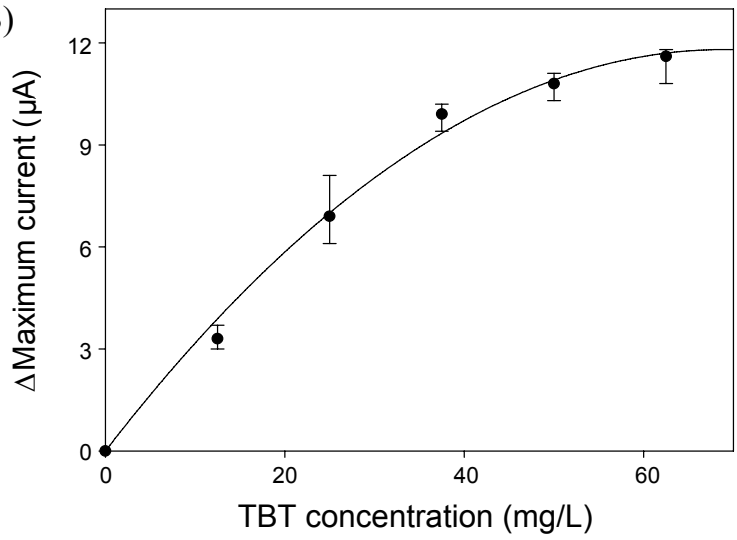

Figure 3. (A) ASV analysis of the bacterial culture media containing various concentrations of TBT (the numbers in the figure indicate the concentrations of TBT in the medium, $\mathrm{mg} / \mathrm{L}$ ). (B) Calibration curve for the analysis of TBT concentrations based on ASV analysis (bars show the standard deviations).

negative potential range than in previous investigations (e.g. $-0.65 \mathrm{~V}$ ). It was interesting to note that when the TBT was dissolved in distilled water, the maximum current was observed in a range of -0.6 to $-0.7 \mathrm{~V}$ (data not shown). This potential shift is probably attributable to the differences in the nature of the samples, which were themselves attributable to the presence of unspecified components in the nutrient broth.

For the quantitative analysis of TBT, the difference in the maximum peak currents between the baseline (i.e. $0 \mathrm{mg} / \mathrm{L}$ of TBT) and TBT measurement trace (TBT containing sample) were measured. Using media containing various concentrations of TBT, the ASV for tin concentration analysis was established. Measurement of the maximum current change after additions of the media containing known concentrations of TBT to the system revealed an increase in the maximum reduction current with increasing concentrations of TBT (Fig. 3B). When the strain TDB-1 culture supernatants of the TDB-1 strain $(30 \mathrm{~h}$ cultivation) were applied to the ASV analysis, an approximate $48 \%$ decrease in TBT concentration was noted (the average concentrations of TBT in the medium varied from $50 \mathrm{mg} / \mathrm{L}$ (before cultivation) to $24 \mathrm{mg} / \mathrm{L}$ (after cultivation)). These results demonstrated that the isolated bacteria, TDB-1, has the ability to reduce the concentration of TBT in a given medium. The GC-FPD data also confirmed the TBT degradation activity of the bacteria (Table 1). After $30 \mathrm{~h}$ of cultivation, the GC-FPD results revealed an approximate $54 \%$ reduction in the concentration of TBT in the culture medium. In the initial cultivation
Table 1. GC-FPD analysis of the organotin concentrations in the strain TDB-1 cultured media

\begin{tabular}{cccc}
\hline \multirow{2}{*}{ Samples } & \multicolumn{3}{c}{$\mathrm{mg} / \mathrm{L}$ concentration (and standard deviations) } \\
\cline { 2 - 4 } & $\mathrm{TBT}$ & $\mathrm{DBT}$ & $\mathrm{MBT}$ \\
\hline Before cultivation & $54(4.5)$ & $\mathrm{ND}$ & $\mathrm{ND}$ \\
After cultivation & $25(2.8)$ & $0.3(<0.1)$ & $0.5(<0.1)$ \\
\hline
\end{tabular}

$\mathrm{ND}=$ not detectable

stage, no DBT and MBT were detected in the medium. After $30 \mathrm{~h}$ of cultivation, however, substantial amounts of TBT degradation products, including DBT $(0.3 \mathrm{mg} / \mathrm{L})$ and MBT $(0.5$ $\mathrm{mg} / \mathrm{L}$ ) were observed. In previous research, the bacterial degradation of TBT to DBT or MBT has also been demonstrated, and the degradation has been shown to involve the sequential removal of organic groups from the tin atom, which are based principally on biological processes. ${ }^{1,8}$ Therefore, it appears plausible that the formations of DBT and MBT from the medium were predicated on bacterial TBT degradation activity. The observed stoichiometric imbalance in the TBT to DBT and/or MBT conversions appears to be the result of the biosorption of TBT on the cell surface and its accumulation within the cell. ${ }^{1,10}$

In this study, we isolated the TBT resistance bacterial strain, and the TBT degradation activity was evaluated via an electrochemical method. The most troublesome issue pertinent to environmental hormone studies involving TBT is the lack of an effective and rapid analysis method. Therefore, the application of ASV to the TBT analysis may prove to be both simpler and more rapid than GC-FPD. However, it should also be noted that the TBT analytical method employed herein only provides the total tin concentration in a filtered bacteria-free culture medium, principally as the result of the unpredictable complexation of bacterial debris with tin and tropolone, which induces the prompt contamination of electrodes (result not shown). This, therefore, suggests that our electrochemical method might prove effective in distinguishing between TBT-degrading bacteria and non-degrading bacteria, a conclusion supported by the results regarding TBT degradation during the cultivation of the TBD-1 strain. Further studies are warranted to establish both the physiological characteristics of the bacterial strains and the efficacy of the electrochemical TBT analysis method under varying experimental conditions, including differing preconcentration conditions with different scanning ranges, using different electrode materials.

Acknowledgments. This paper was supported by Konkuk University in 2010.

\section{References}

1. Gadd, G. M. Sci. Total Environ. 2000, 258, 119.

2. Dubey, S. K.; Roy, U. Appl. Organometal. Chem. 2003, 17, 3.

3. Harino, H.; O'hara, S. C. M.; Burt, G. R.; Pope, N. D.; Chesman, B. S.; Langston, W. J. J. Mar. Biol. Ass. U.K. 2002, 82, 893.

4. Boutakhrit, K.; Quarin, G; Ozkan, S. A.; Kauffmann, J. M. Electroanal. 1996, 8, 789 .

5. Heppeler, F.; Sander, S.; Henze, G. Anal. Chim. Acta 1996, 319, 19.

6. Demkin, A. J. Anal. Chem. 2006, 61, 153.

7. Kawai, S.; Kurokawa, Y.; Harino, H.; Fukushima, M. Environ. Pollut. 1998, 102, 259

8. Bernat, P.; Dlugonski, J. Chemosphere 2006, 62, 3.

9. Pain, A.; Cooney, J. J. Arch. Environ. Con. Tox. 1998, 35, 412.

10. Gadd, G. M.; Gray, D. J.; Newby, P. J. Appl. Microbiol. Biotechnol. 1990, 34, 116. 